\title{
THEOREMS CONCERNING POSITIVE DEFINITIONS OF FINITE ASSEMBLAGE AND INFINITE ASSEMBLAGE
}

\author{
BY MR. C. J. KEYSER.
}

(Read before the American Mathematical Society, December 28, 1900.)

THE well known wide disagreement* among workers in assemblage theory as to the meaning that should be attached to certain indispensable terms, such as assemblage, defined assemblage, given assemblage, law of formation, and so on, indicates alike the need and the possibility of a more critical grounding of this doctrine. Here as elsewhere the guiding principle of criticism should be the principle of Parsimony, the so-called Razor of Occam : Entianon sunt multiplicanda præter necessitatem. For the problem is one of simplification, of logical economy, of minimizing hypothesis. The ideal is, in a word, to dispense with the undefined. Now nothing seems clearer than that no amount of criticism, however acute, can completely eliminate the undefined from the foundations of knowledge. For every explicit involves some implicit, and so there must be not merely assumption, but implicit and therefore undefined assumption. Whence appears that a residuum of indetermination and doubt must elude even the most refined and searching analysis, and ultimate simplicity, perfect certainty, absolute rigor, remain beautiful dreams, destined never to be quite realized. Nevertheless the problem admits of closer and closer approximate solution, which, theoretically at least, takes the form of an unending series, namely, of substitutions of simpler (there is no simplest) undefined for the less simple. Unfortunately the process appears to conduct sooner or later from seeming light and certainty into the "frightful shadow-land of metaphysics." But it is only appearance and only seeming, for it is the common lot, consciously or unconsciously. to dwell in that land always. Shadows critical reflection undoubtedly produces, but shadows are incident to the illumination of darkness. Cousin is right : La critique est la vie de la science.

* Cf. J. Tannery : "De l'infini mathématique," Revue générale des sciences, vol. 8 (1897). Dedekind : Was Sind und Was Sollen die Zahlen. \& 3. Art. 32 ; \& 5, Art. 64 . G. Cantor: "Sur les ensembles infinis et linéaires de points," Acta Math., vol. 2. Couturat : De l'infini mathématique, Appendice, Note IV. E. Borel : Leçons sur la théorie des fonctions, Chap. I. J. Tannery : Introduction à la théorie des fonctions d'une variable, \& 15 . 
This paper presents some results of an attempt, consistently with the mentioned principle of criticism, to show that definitions, of a more general character than those usually considered to be mathematically available, are in fact sufficient for the establishment of certain fundamental propositions of assemblage theory.

\section{Hypotheses, and Definition of Terms.}

It is assumed that, if there be anything which throughout some definite time is intrinsically regardable as an individual, as one, this intrinsic capability persists throughout every definite time. Any such thing will be named element. It is further assumed: (1) that there are elements ; (2) that one of the elements is the idea $\varepsilon$, that each of the elements, including $\varepsilon$, is an element; (3) that, if two elements be distinguishable, the mark of distinction is an element.

The element $\varepsilon$, serving as a bond of union of all elements, strictly defining all as an individual, as the sole invariant content of the element category, imparts sufficient precision to the expression totality of elements. This totality, which might be called the universe of elements, may be denoted by $U$. $U$ being, by definition, itself an element, is to be regarded as contained in itself.

Two elements are identical if they are indistinguishable from each other. Two such elements are, for all logical purposes, for attention, not two but one ; indeed, they can not even be counted as two, for to suppose them so countable is to suppose them capable of arrangement in the timeorder of before and after, which is tantamount to supposing them distinguishable. Hence $U$ contains no identical elements : there are no identical elements.

An element that is two or more elements regardable as an individual may be called a composite element; in the contrary case, an ultimate or simple element.

Now let $e$ and $e^{\prime}$ be any two elements. There is at least one mark of distinction. Represent it by the prime'. This mark is, by (3), itself an element. Consequently $e^{\prime}$ is a composite element. Similarly for $e$. Like reasoning applied to the element' will show it to be composite, and so on indefinitely ; indefinitely, for if the process exemplified could terminate, it would end with an element having no mark of distinction, $i$. e., with a mark of distinction itself without distinction. Such a mark would be an element identical with some (in fact every) other. Hence all elements are composite: there are no simple or ultimate elements. 
It thus appears that an element is a shield with two sides, one fact $F$ with two phases : the phase of being constituted of elements, constituents; and the phase of being a bond uniting the latter into one, an individual. The two phases of $F$ may be briefly called respectively the multi-phase and the $u n i$-phase. Neither is logically prior. Either may be regarded as primary, the other as secondary - the choice to be determined by considerations of expedience. $F$ will be called element or assemblage (of elements) according as the uni-phase or the multi-phase of $F$ be taken as primary. The notions, element and assemblage, are, therefore, reciprocal* and complemental, each is implicit in, incomplete without, determined by, the other.

This principle of reciprocity enables us to say : all elements and assemblages are defined. If $F$ be an assemblage, its definition is its uni-phase, its element-character ; if $F$ be thought as an element, its definition is its multi-phase, its character as a certain composite. For easier comparison of this with the corresponding conceptions of Cantor and Dedekind, $\dagger$ we state it at length thus : Every assemblage is defined in the sense that the principle of excluded middle validates, with respect to any assemblage, the proposition : any element whatever is a constituent of the assemblage or it is not. Reciprocally, every element is defined in the sense that the same principle justifies, in regard to any element, the proposition : the constituents of any assemblage whatever compose the element or they do not.

The term "defined" is here used in its objective, which is at the same time its fundamental and etymological, sense of bounded or marked off from. Every such objective definition is an $F$ with its two phases. Its essence is being, not actually regarded but merely regardable. When actually regarded, made the recipient of attention, then, lying in consciousness or expressed in language or both, it becomes an ordinary, subjective, definition. When so expressed the corresponding $F$ (assemblage or element) may be said to be given. Discussion of the exceeding difficult question \$ of "given" is reserved for a future communication as is also a fuller exhibition of the cardinal importance of the principle of recipro-

* It is noteworthy that the so-called " principle of reciprocity" which manifests itself familiarly in various specialized and concrete ways, notably in geometry, is in all strictness a principle and is such for assemhlage theory in general, being inherent, as just shown, in the inmost core of the discipline.

† Cf. Cantor and Dedekind : Op. cit.

$\ddagger$ In relation to this matter, the first of J. Tannery's essays already cited may be consulted with much profit. 
city. In regard to the latter we merely add here the now rather obvious facts : that assumptions (1), (2), and (3) involve like assumptions respecting assemblages; that there are no identical assemblages ; that, as every assemblage has its uni- and every element its multi-phase, there is one and but one element for each assemblage and reciprocally ; and, that, in particular, $U$ plays the double rôle of being at once the totality of elements and the totality of assemblages, including itself in both characters.

If $E$ and $E^{\prime}$ are any two assemblages such that there is an assemblage $M$ having the properties: (1) each element of $M$ is a pair of elements $e$ and $e^{\prime}$, where $e$ and $e^{\prime}$ signify element of $E$ and element of $E^{\prime}$ respectively ; (2) each element $e$ occurs once and but once, and no element $e^{\prime}$ occurs twice, in $M$; the assemblage $E$ may be said to be projectible upon the assemblage $E^{\prime}$. As in $M$ each element $e$ of $E$ is associated with a certain element $e^{\prime}$ of $E^{\prime}$, the assemblage $M$ may be said to indicate a mode or manner of the projectibility; $a$ rather than the manner, because obviously, if $E$ be projectible upon $E^{\prime}$ in one manner, it will in general be projectible upon $E^{\prime}$ in more than one manner. If, in a given $M, e_{k}^{\prime}$ of $E^{\prime}$ is associated with $e_{k}$ of $E, e_{k}{ }^{\prime}$ may be called the projection of $e_{k}$ for the given $M$. The totality of such projections is an assemblage which may be denoted by $P$.

If two assemblages $E$ and $E^{\prime}$ are each projectible upon the other in a manner $M, E$ and $E^{\prime}$ may be said to be congruent in the manner $M$, or a unique and reciprocal correspondence may be said to exist in the manner $M$ between $E$ and $E^{\prime}$. If two assemblages $E$ and $E^{\prime}$ be congruent, the relationship may be stated symbolically by writing

$$
E \equiv E^{\prime} \text {. }
$$

If every element of an assemblage $A$ is an element of an assemblage $A^{\prime}$ and if one or more elements of $A^{\prime}$ are not in $A$, $A$ will be called a part (partie intégrante of Cantor, echter Theil of Dedekind) of $A^{\prime}$, and we may write

$$
A<A^{\prime} \text {. }
$$

If this relation subsists between $A$ and $A^{\prime}$, then plainly $A$ is projectible upon $A^{\prime}$. Also any $E$ is congruent with itself. The equation

$$
A_{1}+A_{2}=A
$$

may be employed to indicate at once that $A_{1}$ and $A_{2}$ are each a part of $A$, that $A_{1}$ and $A_{2}$ have no element in common, and that every element of $A$ is an element of $A_{1}$ or of $A_{2}$. 


\section{Demonstration of Theorems.}

Let $E$ be an assemblage. Either $E$ is congruent with an assemblage

$$
1,2, \cdots, n,
$$

where $n$ is some definite integer, or such is not the case. If $E$ be not congruent with $(N)$, the assemblage

$$
1,2, \cdots, n, \cdots
$$

is projectible upon $E$. To prove this it is sufficient to prove that, however large a value $n^{\prime}$ of $n$ be chosen in advance, the assemblage

$$
1,2, \cdots, n^{\prime}, n^{\prime}+1
$$

is projectible upon $E$. That this assemblage is projectible upon $E$ follows immediately from the fact that, were it not so projectible, $E$ would itself be projectible upon $(N)$ for $n \equiv n^{\prime}$, which is contrary to hypothesis. Let the assemblage

$$
\left(1, e_{1}\right),\left(2, e_{2}\right), \cdots,\left(n, e_{n}\right), \cdots
$$

indicate a manner of projectibility of $\left(N^{\prime}\right)$ upon $E$. Then the assemblage $P$ of projections will be

and we may write

$$
e_{1}, e_{2}, \cdots, e_{n}, \cdots
$$

$$
E=P+R
$$

where $R$ denotes the assemblage of those elements of $E$ which, if there be any such, are not elements of $P$. Represent the assemblage

$$
e_{2}, e_{4}, \cdots, e_{2 n}, \cdots
$$

by $P_{1}$. It is plain that $P$ is projectible upon $P_{1}$ in the manner

$$
\left(M_{1}\right) \quad\left(e_{1}, e_{2}\right),\left(e_{2}, e_{4}\right), \cdots,\left(e_{n}, e_{2 n}\right), \cdots
$$

and $R$ is projectible upon itself; hence the assemblage $P+R$ is projectible upon the assemblage $P_{1}+R$; but

whence

$$
P+R=E \text { and } P_{1}<P
$$

$$
P_{1}+R<E \text {. }
$$

$\boldsymbol{E}$ is therefore projectible upon a part of itself. As any assemblage is obviously congruent with the assemblage of its projections, $E$ is seen to be congruent with a part of itself. Accordingly we may state the 
Theorem ( $\alpha)$. If any assemblage $E$ be such that there is no definite value of $n$ for which the assemblage

$$
1,2, \cdots, n
$$

is congruent with $E$, then $E$ is congruent with a part of itself.

From this follows directly the contrapositive

Theorem $(b)$. If any assemblage $E$ be not congruent with a part of itself, then, for some definite value of $n$, the assemblage

is congruent with $E$.

$$
1,2, \cdots, n
$$
that

Are the converses of these propositions correct? Suppose

$$
A<E \text {, and that } E \equiv A \text { in some manner } M \text {. }
$$

It is required to determine whether there is a determinate integer $n$ such that the assemblage

$$
1,2, \cdots, n
$$

is congruent with $E$.

Let $e_{k}$ denote any definite element of $E$. In $M$ this element is paired with some definite element $e_{j}$ of $E$, whence $e_{j}$ is an element of $A$. Now, if $E$ is congruent with $(N)$ in one or more manners, let $M^{\prime}$ stand for any definite one of them. In $M^{\prime}$ the element $e_{k}$ has for its projection, or is paired with, some definite integer $n^{\prime}$ of $(N)$ where of course it is undetermined whether $n^{\prime}$ is less than or equal to $n$. Accordingly, $M$ and $M^{\prime}$ together furnish a scheme for associating in a one to one fashion the elements of $A$ with those of $(N)$ : given any element, as $e_{k}$ of $E$, those two elements, $e_{j}$ and $n^{\prime}$, of $A$ and $(N)$, respectively, shall be associated, which are associated with $e_{k}$ in $M$ and $M^{\prime}$, respectively. Denote by $M^{\prime \prime}$ the assemblage of such pairs $e_{j}, n^{\prime}$. It thus appears that if

$$
E \equiv A
$$

in some definite manner $M^{\prime}$, then

$$
A \equiv(N)
$$

in a definite related manner $M^{\prime \prime}$. But since

$$
A<E \text {, }
$$

there is at least one element $e_{i}$ of $E$ which is not an element of $A$, and, therefore, no one of such elements $e_{i}$ enters a pair in $M^{\prime \prime}$. Hence $A$ is not congruent with $(N)$ in the 
manner $M^{\prime \prime}$; whence follows that $E$ is not congruent with $A$ in the manner $M^{\prime}$, and, therefore, not in any manner.

We have, accordingly, the following propositions:

Theorem $\left(a^{\prime}\right)$. If any assemblage $E$ is congruent with a part of itself, there is no assemblage

$$
1,2, \cdots, n
$$

with which $E$ is congruent.

Theorem $\left(b^{\prime}\right)$. If there is an assemblage

$$
1,2, \cdots, n
$$

with which an assemblage $E$ is congruent, $E$ is not congruent with a part of itself.

These reciprocally contrapositive propositions are the converses, respectively, of $(a)$ and $(b)$.

\section{Logical Significance of the Four Theorems.}

Denote by $U$ the assemblage of all assemblages, including $U$. The elements of $U$ are the component assemblages of $U$. Of these there are two, $F_{1}$ and $I_{1}$, such that the former signifies the assemblage of all assemblages which are not, and the latter signifies the assemblage of all assemblages which are congruent each with a part of itself. By the principle of excluded middle,

By the same principle,

$$
U=F_{1}+I_{1}
$$

$$
U=F^{\prime}+I^{\prime}
$$

where $F^{\prime}$ denotes the assemblage of all assemblages of which each has, and $I^{\prime}$ denotes the assemblage of all assemblages of which none has, the property of being projectible upon (or congruent with) an assemblage

$$
1,2, \cdots, n \quad \text { ( } n \text { definite). }
$$

Now the logical import of the propositions $(a),(b),\left(a^{\prime}\right)$, and $\left(b^{\prime}\right)$ is that $F_{1}$ and $F^{\prime}$, as also $I_{1}$ and $I^{\prime}$, are identical, element for element. Accordingly, we may define as follows :

(1) A finite assemblage is one which is not congruent with any part of itself.

(2) An infinite assemblage is one which is not congruent with any assemblage.

$$
1,2, \cdots, n \quad(n \text { definite }) .
$$

Or quite equivalently thus : 
(3) A finite assemblage is one which is congruent with some assemblage

$$
1,2, \cdots, n \quad \text { ( } n \text { definite). }
$$

(4) An infinite assemblage is one which is congruent with a part of itself.

In neither pair of definitions are the notions finite and infinite formally represented as mutually exclusive or as negatives of each other ; while the second pair has the additional advantage of representing both the notions in question as positive $*$ concepts.

Before passing to the final section of this paper, it seems worth while to pause long enough to glance, from the present point of view, at the notion, cardinal number of a finite assemblage, and to offer a proof that this notion, for any given finite assemblage, possesses the property of invariance.

Consider the ordered assemblage

$$
1,2, \cdots, n^{\prime}, \cdots, n, \cdots n^{\prime \prime},
$$

where $n^{\prime}, n$ and $n^{\prime \prime}$ are definite and subject to the condition that, in $(S), n^{\prime}$ comes before $n$ and the latter before $n^{\prime \prime}$. Denote by $S_{n^{\prime}}, S_{n}$, and $S_{n^{\prime \prime}}$ the assemblages obtained by supposing in succession that $(S)$ ends with $n^{\prime}, n$ and $n^{\prime \prime}$, and in each case abstracting from the order of the elements. $S_{n^{\prime}}, S_{n}$, and $S_{n^{\prime \prime}}$, being congruent each with itself, are finite assemblages, and, as by definition

we have by (3)

$$
S_{n^{\prime}}<S_{n} \text { and } S_{n}<S_{n^{\prime \prime}}
$$

$$
\text { neither } S_{n^{\prime}} \equiv S_{n} \text { nor } S_{n} \equiv S_{n^{\prime \prime}} \text {. }
$$

Now let $E$ be any finite assemblage. By (3) there is some $S_{n}$ for which

$$
E \equiv S_{n}
$$

We now define $n$ to be the cardinal number of the assemblage $E$. Plainly, this number is independent of every arrangement of the elements of $E$; for if, by any particular arrangement,

$$
E \equiv S_{n^{\prime}} \text { or } E \equiv S_{n^{\prime \prime}}
$$

we would have also

* As to this phase of the matter and also in relation to definitions (1) and (4), cf. Jules Tannery, op. cit.; Dedekind, op. cit.; G. Cantor, op. cit.; Couturat, op. cit. 


$$
S_{n} \equiv S_{n^{\prime}} \text { or } S_{n^{\prime \prime}} \equiv S_{n^{\prime}}
$$

It readily follows that any two congruent finite assemblages have the same cardinal number.

\section{Connection with the Logic of Relatives: Another Point of View.}

Consider the argument :

Every element of an assemblage $E$ is projected upon some other element of $E$;

No element of $E$ is the projection of more than one element of $E$;

$\therefore \quad$ Every element of $E$ is a projection of an element of $E ; i$. e., the assemblage of projections is congruent with $E$.

This argument is of the type of the so-called syllogism of transposed quantity, a mode of ratiocination of which the earliest scientific account is found in the Logic of De Morgan. Its uses and limitations have been pointed out by Mr. C. S. Peirce, * to whom is due also its perfection as an instrument of research as well as the indication of its significance in modern logic as such. From definition (1) or from the last proposition of section III, it is seen that the conclusion is warranted by the premises always when and only when $E$ is a finite assemblage. Reversing the order of ideas, the above argument, regarded as purely a form, may be taken as the 'logical' discriminant of the assemblage

$$
U=F_{1}+I_{1}
$$

and accordingly we might define : any given assemblage is finite or infinite, belongs to $F_{1}$ or to $I_{1}$, according as the syllogism is valid or not valid for that assemblage. Such appears to be in fact Mr. Peirce's point of view in one or two instances. At all events, it requires merely verbal substitution to render the syllogism in question quite identical with the "Hottentot" and "Texan" arguments employed by him in the works cited below. The interest here attaches, of course, to the demonstrated ultimate equivalence of the two points of view.

Columbia UNIVersity.

* Peirce: "On the Algebra of Logic," Amer. Jour. of Math., vol. 7, p. 201. "On the Logic of Number," Amer. Jour. of Math., vol. 4, p. 75. Definition of "finite," Century Dictionary. 